Review

\title{
Large Intervening Non-Coding RNA HOTAIR Is an Indicator of Poor Prognosis and a Therapeutic Target in Human Cancers
}

\section{Yanlan Yao ${ }^{1}$, Jinming $\mathrm{Li}^{2, \dagger, *}$ and Lunan Wang ${ }^{2, \dagger, *}$}

1 National Center for Clinical Laboratories, Graduate School of Peking Union Medical College, Beijing Hospital of the Ministry of Health, No. 1 Dahua Road, Beijing 100083, China; E-Mail: yaoylan@126.com

2 National Center for Clinical Laboratories, Beijing Hospital of the Ministry of Health, No. 1 Dahua Road, Beijing 100083, China

$\dagger$ These authors contributed equally to this work.

* Authors to whom correspondence should be addressed; E-Mails: jmli63hn@gmail.com (J.L.); lnwang@nccl.org.cn (L.W.); Tel.: +86-10-5811-5053 (J.L.); Fax: +86-10-6521-2064 (J.L.).

External Editor: William Cho

Received: 25 June 2014; in revised form: 26 September 2014 / Accepted: 1 October 2014 / Published: 20 October 2014

\begin{abstract}
In the human genome, the fraction of protein-coding genes that are stably transcribed is only up to $2 \%$, with the remaining numerous RNAs having no protein-coding function. These non-coding RNAs (ncRNAs) have received considerable attention in cancer research in recent years. Breakthroughs have been made in understanding microRNAs and small interfering RNAs, but larger RNAs such as long ncRNAs (lncRNAs) remain an enigma. One lncRNA, HOX antisense intergenic RNA (HOTAIR), has been shown to be dysregulated in many types of cancer, including breast cancer, colorectal cancer, and hepatoma. HOTAIR functions as a regulatory molecule in a wide variety of biological processes. However, its mechanism of action has not been clearly elucidated. It is widely believed that HOTAIR mediates chromosomal remodeling and coordinates with polycomb repressive complex 2 (PRC2) to regulate gene expression. Further study of HOTAIR-related pathways and the role of HOTAIR in tumorigenesis and tumor progression may identify new treatment targets. In this review, we will focus on the characteristics of HOTAIR, as well as data pertaining to its mechanism and its association with cancers.
\end{abstract}


Keywords: HOTAIR; lncrna; polycomb repressive complex 2; prognosis; therapeutic target

\section{Introduction}

In the human genome, the fraction of protein-coding genes that are stably transcribed is only up to $2 \%$ [1]. Thus, a large number of transcripts do not encode proteins. These non-coding RNAs (ncRNAs) can be divided into housekeeping non-coding RNAs and regulatory non-coding RNAs. Housekeeping non-coding RNAs include ribosomal, transfer, small nuclear, and small nucleolar RNAs. They are usually constitutively expressed. The short regulatory ncRNAs $(<200 \mathrm{nt})$ include microRNAs, small interfering RNAs (siRNAs), and piwi-associated RNAs. In addition, long non-coding RNAs (lncRNAs) [2,3], 200-100,000 nt in length, contribute to the regulation of gene expression at various levels, including chromatin modification, transcription, and post-transcriptional processing. Studies have shown that some lncRNAs are dysregulated in various cancers, contributing to tumorigenesis and tumor progression. They may have theranostic applications in human cancers. Among them, the lncRNA HOX antisense intergenic RNA (HOTAIR) was identified in 2007 [4], and numerous other lncRNAs have been discovered by next-generation sequencing and computational prediction. In this review, we summarize recent research on HOTAIR IncRNA, with a focus on its identification, characterization, mechanism of action, and association with related human cancers.

\section{Mechanism of IncRNA Function and IncRNA-Related Cancers}

\subsection{Possible Origins of lncRNAs}

Five kinds of possible origins of lncRNA are shown (Figure 1). First, a protein-coding gene (left, pink) acquires frame disruptions and is transformed into a functional noncoding RNA (right, blue) that incorporates some previous coding sequence. The Xist lncRNA originated by undergoing a metamorphosis from a previous protein-coding gene while incorporating transposable element sequence (Figure 1A); Secondly, following a chromosome's rearrangement, two un-transcribed and previously well-separated sequence regions are juxtaposed and give rise to a multi-exon noncoding RNA. A dog noncoding RNA (supported by ESTs BM537447, C0597044, and DN744681) appears to have arisen following such a lineage-specific change (Figure 1B); Thirdly, duplication of a noncoding gene by retrotransposition generates either a functional noncoding retrogene or a nonfunctional noncoding retropseudo-gene (Figure 1C); Fourthly, Neighboring repeats within a noncoding RNA have their origins in two tandem duplication events (Figure 1D); Fifthly, insertion of a transposable element (yellow) gives rise to a functional noncoding RNA (Figure 1E) [4].

\subsection{Mechanism of IncRNA Function}

Recent studies have shown that lncRNAs participate in various regulatory processes. Figure 2 outlines eight paradigms for how lncRNAs function. 
Figure 1. Possible origins of lncRNA. (A) acquire frame disruptions and transform into a functional noncoding RNA; (B) two untranscribed and previously well-separated sequence regions are juxtaposed through chromosome rearrangement; (C) generates either a functional noncoding retrogene or a nonfunctional noncoding retropseudo-gene, resulting from duplication of a noncoding gene by retrotransposition; (D) to occur neighboring repeats; (E) by inserting a transposable element.

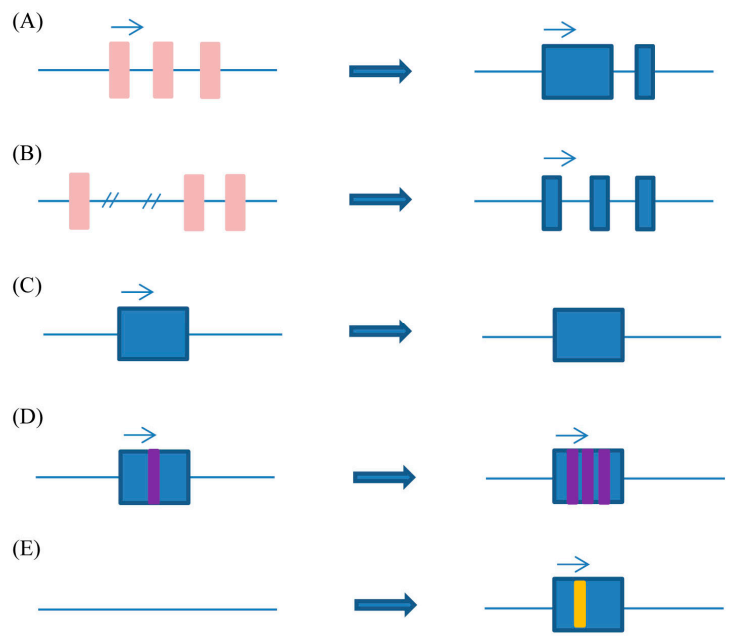

Figure 2. Possible mechanisms of lncRNA function. lncRNAs can (1) interfere with downstream gene transcription by inhibiting RNA polymerase II recruitment or (2) promote downstream gene expression by inducing chromatin remodeling and histone modifications; (3) An antisense lncRNA can modulate alternative splicing patterns by hybridizing to the complementary transcript; (4) Hybridization of lncRNA and mRNA allows Dicer to generate endogenous siRNAs; lncRNAs can bind to protein partners to (5) modulate protein activity; (6) serve as structural components; or (7) alter protein localization; (8) lncRNAs can generate small RNA precursors through certain processes.

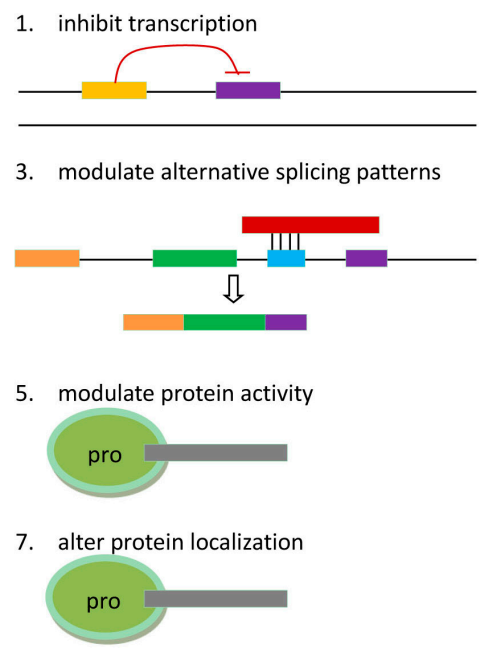

2. promote transcription

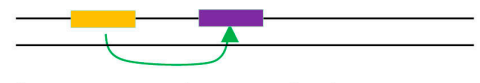

4. generate endogenous siRNAs

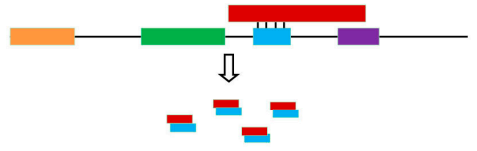

6. form RNA-protein complexes

pro

8. generate small RNA precursors

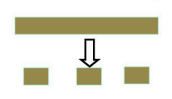

\subsection{Cancer-Related lncRNAs.}

The evidence for IncRNA involvement in tumorigenesis includes findings that the lncRNA MALATI (metastasis-associated lung adenocarcinoma transcript 1) promotes lung cancer metastasis [5]; 
participates in regulation of cell proliferation, migration, and invasion in colorectal cancer metastasis [6,7]; and is associated with an increased risk of liver tumor recurrence [8,9], and breast cancer metastasis [10].

HOTAIR is overexpressed in breast, colorectal, liver, and nasopharyngeal cancers [11-15]. Loss of HOTAIR moderates the invasiveness of breast cancer, particularly in cells in which polycomb repressive complex 2 (PRC2) is up-regulated. Similarly, HOTAIR also induces genome-wide PRC2 retargeting, participates in epigenetic regulation in colorectal cancer, and promotes metastases. In liver cancer, HOTAIR is a prognostic factor of hepatoma metastases and relapse after hepatic resection or liver transplantation. Moreover, up-regulated expression of HOTAIR is an indicator of poor prognosis in many other kinds of cancers [8,16-21].

Another lncRNA, highly up-regulated liver cancer $(H U L C)$, is reportedly overexpressed in hepatocellular carcinoma, other metastatic liver cancers [22,23] and liver cancer cell lines [24]. Up-regulated HULC may act as miRNA sponge and down-regulate $m i R-372$, an important miRNA in liver cancer. This results in the phosphorylation of cAMP responsive element binding protein 1 (CREB1). Moreover, phosphorylated CREB1 participates in transcriptional inducement of $H U L C$ in the nucleus since it contains a CREB binding site in the core promoter. In other words, fine tuning of $H U L C$ expression is part of an auto-regulatory loop [24]. In addition to $H U L C$, another lncRNA transcribed from gene $H 19$ promotes tumor growth in hepatocellular carcinoma [25].

$A N R I L$ (antisense non-coding RNA in the INK4 locus) was first identified through genetic analysis of familial melanoma patients [26]. ANRIL was also found to be associated with breast cancer [27-29], nasopharyngeal carcinoma, basal cell carcinoma, and glioma. Several reports have provided mechanistic insights into its molecular functions, which include binding to SUZ12 and recruitment of PRC2, but further studies are needed. Table 1 lists several cancer-associated lncRNAs and their functions.

Table 1. Cancer-related lncRNAs.

\begin{tabular}{cccc}
\hline Cancer Type & IncRNA & Function & Reference \\
\hline \multirow{3}{*}{ Breast cancer } & HOTAIR & Chromatin remodeling & {$[11,30]$} \\
& ANRIL & Chromatin remodeling & {$[27-29]$} \\
& MALAT1 & Gene relocation, scaffold, alternative splicing & {$[4,10]$} \\
\hline \multirow{2}{*}{ Colorectal } & HOTAIR & Chromatin remodeling & {$[11,13]$} \\
cancer & HULC & Liver metastasis & {$[23]$} \\
& MALAT1 & Gene relocation, scaffold, alternative splicing & {$[7]$} \\
\hline \multirow{2}{*}{ Hepatocellular } & MALAT1 & Gene relocation, scaffold, alternative splicing & {$[4]$} \\
carcinoma & HULC & MiRNA sponge & {$[23]$} \\
& HOTAIR & Chromatin remodeling & {$[31]$} \\
& $H 19 \operatorname{lncRNA}$ & Promoting tumor growth & {$[25]$} \\
\hline
\end{tabular}

\section{Characteristics and Mechanisms of HOTAIR}

\subsection{Identification and Characterization of HOTAIR}

HOTAIR has attracted considerable attention from researchers worldwide. It was found to promote breast cancer metastasis and then shown to be pervasively overexpressed in most human cancers when 
tumor tissue was compared with adjacent noncancerous tissue. HOTAIR is a $2158 \mathrm{nt} \operatorname{lncRNA}$ discovered by Howard Chang's group (lncRNA Database, http://www.lncrnadb.org/). An ultra-high-density HOX tiling array analysis showed that over 200 ncRNAs are transcribed from the four human $H O X$ loci (HOXA-HOXD). HOTAIR is transcribed from the developmental HOXC locus located at chromosome 12q13.13, in a position intergenic and antisense to the flanking $H O X C 11$ and $H O X C 12$ genes [4,13,32,33]. HOTAIR induces the transcriptional silencing of the HOXD locus on chromosome 2 in trans [32,34]. It is the first lncRNA found to regulate gene expression in trans. Overexpression of HOTAIR has been found in breast cancer, colorectal cancer, and hepatoma.

Sequence analyses suggested that orthologues of HOTAIR are present only in mammals [33]. HOTAIR is located in the HOXC locus, but it has evolved faster than the neighboring HOXC genes. So, HOTAIR has poorly conserved sequences and considerably conserved structures. The cognate murine HOTAIR shows only $58 \%$ sequence identity to human HOTAIR. Deleting the HOXC cluster containing HOTAIR in mice produced no obvious effect and did not affect HOXD10 expression, which suggests poor conservation of function [35]. However, a 239-bp domain within the 1804-bp exon 6 is well conserved and could be essential for HOTAIR function [33].

\subsection{Mechanisms of HOTAIR Function}

HOTAIR interacts with and recruits PRC2 and regulates the chromosome occupancy of EZH2 (a subunit of PRC2), which leads to histone H3 lysine 27 trimethylation of the HOXD locus. Moreover, HOTAIR can trigger the epithelial-mesenchymal transition (EMT) and interact with other small RNAs.

\subsubsection{HOTAIR Reprograms the Chromatin State through PRC2/LSD1}

HOTAIR is the first lncRNA that has been found to act in trans. It is transcribed from HOXC but mediates the silencing of some tumor-suppressing genes of the HOXD locus. It is widely believed that PRC2, which comprises the EZH2 (Enhancer of Zeste 2, a histone methylase), SUZ12, and EED subunits, is essential for this silencing. HOTAIR recruits and interacts with PRC2 and regulates chromosome occupancy by EZH2 or SUZ12 [34] through its 5' domain, which leads to histone H3K27 methylation and chromosomal remodeling of the HOXD locus. In other words, HOTAIR induces genome-wide re-targeting of PRC2 and an altered gene expression pattern, promoting tumorigenesis and tumor progression [4,11,21,33], as described in Figure 2(7). Enforced HOTAIR expression in epithelial cancer cells increases invasive and metastatic abilities and reprograms the PRC2 occupancy pattern to resemble that of embryonic fibroblasts. Furthermore, HOTAIR function is not just limited to HOXD; it can recruit PRC2 to 854 genes that do not bind with PRC2 under normal conditions, resulting in gene overexpression or repression. Conversely, depletion of PRC2 proteins significantly reverses the influence of HOTAIR, providing another line of evidence that HOTAIR acts by coordinating with PRC2 [11]. As HOTAIR and PRC2 are mutually dependent, drugs targeting endogenous HOTAIR or inhibitors of HOTAIR-PRC2 interactions could serve as a new therapy strategy against tumors overexpressing polycomb proteins. Understanding the precise molecular mechanisms by which HOTAIR regulates PRC2 will be a critical first step in exploring this potential new avenue in cancer therapy. 
The binding of HOTAIR to EZH2 also depends on EZH2 protein modification. EZH2 is phosphorylated by cyclin-dependent kinase 1 (CDK1) at threonine residues 345 and 487 in a cell-cycle-dependent manner. A phosphomimetic mutation at residue T345 was found to increase HOTAIR binding, implying that phosphorylation of EZH2 by CDK1 or CDK2 might enhance its function during the $\mathrm{S}$ and $\mathrm{G} 2$ phases in the cell cycle [34].

In addition to PRC2, the $H O X D$ region is bound by CoREST/REST repressor complexes, which contain lysine-specific demethylase 1 (LSD1). This demethylase mediates enzymatic demethylation of dimethylated H3K4 (H3K4me2). HOTAIR has also been found to bind to the LSD1/CoREST/REST complex [32]. The 5' domain of HOTAIR binds to PRC2, whereas the 3' domain binds to the LSD1 complex. Thus, HOTAIR is a modular bifunctional lncRNA with distinct binding domains for a histone methylase and demethylase, and it serves as a scaffold for at least two distinct histone modification complexes. The modifications of these DNA-binding proteins by HOTAIR regulate global gene expression. The bifunctional role of HOTAIR might be required for coordinating histone modifications of H3K27 methylation and H3K4 demethylation for epigenetic gene silencing to promote metastatic processes [36].

\subsubsection{HOTAIR Triggers the EMT Process}

The role of HOTAIR in the tumorigenesis of epithelial cancers involves EMT triggering and stemness acquisition, and suppression of HOTAIR may reverse the EMT process [37]. Padua Alves et al. [38] found that treatment with TGF- $\beta 1$ increased HOTAIR expression and triggered the EMT program. Interestingly, ablation of HOTAIR expression by siRNA prevented the EMT program stimulated by TGF- $\beta 1$, as well as the colony forming capacity of colon and breast cancer cells. Further, the colon cancer stem cell subpopulation $\left(\mathrm{CD} 133^{+} / \mathrm{CD} 44^{+}\right)$expressed much higher levels of HOTAIR than the non-stem cell subpopulation. However, the mechanism by which HOTAIR triggers the EMT has rarely been elucidated. In gastric cancer cells, the EMT may be triggered by HOTAIR through up-regulation of "snail" (a transcription factor involved in EMT). This is similar to the functional scenario of Figure 2(2) [38]. Certainly, further demonstration is needed for supporting this viewpoint.

\subsubsection{HOTAIR Interacts with Tumor Suppressor miRNAs}

Recent studies suggest that lncRNAs interact with other classes of non-coding RNAs, including microRNAs (miRNAs), and modulate their regulatory activity [39]. IncRNA can serve as an miRNA sponge and interfere with the tumor suppressor function of protective miRNAs, resulting in tumorigenesis. Chiyomaru et al. [36] demonstrated that genistein inhibited prostate cancer ( $\mathrm{PCa}$ ) cell growth by down-regulating oncogenic HOTAIR and its interaction with the tumor suppressor miR-34a. miR-34a was up-regulated by genistein in PCa cells, whereas HOTAIR expression was down-regulated, suggesting that $m i R-34 a$ plays an important role in the effects of genistein treatment. Furthermore, luciferase reporter assays confirmed the binding of $m i R-34 a$ to its predicted binding site in HOTAIR [36], providing further support for their interaction. 


\subsubsection{HOTAIR Competes with BRCA1}

The BRCA1 gene is directly associated with hereditary breast cancer, and its protein product, BRCA1, is normally expressed in the cells of the breast and other tissues. BRCA1 helps repair damaged DNA or destroy the cell if the DNA cannot be repaired. BRCA1 is involved in the repair of chromosomal damage, playing an important role in the error-free repair of DNA double-strand breaks. Moreover, in EZH2, the BRCA1-binding region overlaps with the HOTAIR-binding domain, and BRCA1 inhibits the binding of EZH2 to HOTAIR. Thus, decreased expression of BRCA1 causes genome-wide EZH2 retargeting and elevates H3K27me3 levels at PRC2 target loci, which play a role in HOTAIR-related breast cancers [40], as shown in Figure 2(7).

\section{HOTAIR-Related Cancers}

HOTAIR plays a role in tumorigenesis and tumor progression. In tumorigenesis, HOTAIR acts as an oncogene; in tumor progression, HOTAIR promotes invasion and metastases. However, the pathways and molecules that mediate HOTAIR's effects must be understood before HOTAIR can be used in cancer treatment.

\subsection{HOTAIR Is a Powerful Prognostic Factor in Breast Cancer (BC)}

Dysregulation of HOTAIR in various cancers is associated with metastasis and tumor progression. HOTAIR was found to be highly up-regulated in primary, as well as metastatic, breast tumors, and its elevation correlated with both metastasis and poor survival rate $[11,30]$.

HOTAIR expression is increased in primary breast tumors and metastases (as much as 2000-fold), and HOTAIR expression in primary tumors is a powerful predictor of eventual metastasis and death, independent of known clinicopathologic risk factors. Gupta et al. [11] measured HOTAIR levels in an independent panel of 132 primary breast tumors (stage I and II) with extensive clinical follow-up. Nearly one-third overexpressed HOTAIR by more than 125 -fold when compared to normal breast epithelia. A high level of HOTAIR is a significant predictor of subsequent metastasis $(p=0.0004)$ and death $(p=0.005)$. Stable overexpression of HOTAIR by several hundredfold through retroviral transduction promoted colony growth in soft agar. Conversely, depletion of HOTAIR with siRNA in the MCF7 cell line, which expresses endogenous HOTAIR, decreased matrix invasiveness, thus demonstrating the function of HOTAIR in vitro. In addition, Gupta et al. labeled control and HOTAIR-expressing MDA-MB-231 cells with firefly luciferase and grafted the cells into mammary fat pads. Two weeks later, the number of luciferase foci in the lung fields was higher in mice with HOTAIR-expressing primary tumors than in control mice, which suggests that HOTAIR promotes lung metastasis in vivo.

\subsubsection{HOTAIR and the Methylation Status of Down-Stream CpG Islands}

The prognostic value of HOTAIR in breast cancer was widely accepted until an article published at the end of 2012 challenged the prevailing view. Lu et al. [41] analyzed DNA methylation in 348 primary breast cancer tissues with methylation-specific PCR and found a positive correlation between DNA methylation and HOTAIR expression. Methylation was associated with unfavorable 
disease characteristics. However, no significant associations were found between HOTAIR expression and clinical or pathologic features. Patients with high HOTAIR expression had lower risks of relapse and mortality than those with low HOTAIR expression. These findings suggest that intergenic DNA methylation may be important in regulating HOTAIR expression and that HOTAIR expression may not be an independent prognostic marker in breast cancer. However, the findings require further validation in independent studies.

\subsubsection{HOTAIR Function in Breast Cancer Is Associated with Estrogen Receptor Status}

Recent studies showed that HOTAIR is an independent prognostic factor of metastases in estrogen receptor (ER)-positive primary breast cancer. A retrospective study of 164 primary breast cancer patients by Sørensen et al. [15] found that a high level of HOTAIR was significantly associated with worse prognosis $(p=0.012)$. The association was even stronger when only ER-positive tumor samples were considered $(p=0.0086)$. Bhan et al. [42] demonstrated that HOTAIR requires transcriptional induction by E2. The HOTAIR promoter contains multiple functional estrogen response elements. In the presence of E2, ERs and various ER coregulators bind to the promoter. Meanwhile, the level of H3K4 trimethylation, histone acetylation, and RNA polymerase II recruitment is enriched at the HOTAIR promoter. In contrast, knockdown of ERs down-regulates E2-induced HOTAIR expression. Given these findings, therapeutic methods that target HOTAIR or regulate ER status may help suppress the progression of breast cancer.

\subsection{HOTAIR Is a Potential Prognostic Factor in Gastroenteric Tumors}

As in breast cancer, HOTAIR also induces genome-wide PRC2 retargeting, participates in epigenetic regulation and promotes metastases in colorectal cancer (CRC) $[11,13,43]$. Analysis of approximately 100 cancer tissues showed that HOTAIR expression was significantly higher in cancer tissues than in normal tissue samples $(p=0.002)$. After dividing the cancer tissues into a high HOTAIR expression group $(n=20)$ and a low expression group $(n=80)$ using a HOTAIR/GAPDH ratio of 0.273 , further analysis revealed that the high expression group exhibited less differentiated histology $(p=0.024)$, greater tumor depth $(p=0.039)$, greater liver metastases $(p=0.006)$, and worse prognosis $(p=0.0046)$. Transduction of lentiviral vectors encoding HOTAIR into the HCT116 cell line promoted cell invasion in Matrigel $(p<0.05)$. Conversely, siRNA-induced knockdown of HOTAIR decreased cell invasion $(p<0.05)$, further suggesting that HOTAIR is related to liver metastases and is a potential prognostic factor in colorectal cancer [13]. On the other hand, HOTAIR suppression sensitized cancer cells to tumor necrosis factor $\alpha(\mathrm{TNF}-\alpha)$, induced apoptosis, and rendered the cells more sensitive to the chemotherapeutic agents cisplatin and doxorubicin [13], indicating that HOTAIR could be a target for therapy.

Svoboda M, et al. [43] also assessed the prognostic value of HOTAIR expression. On one hand, they underlined the prognostic potential of HOTAIR expression level in tumor tissues of CRC patients, both in univariate analysis $(p=0.046)$ and multivariate analysis $(p=0.048)$. What is more, they demonstrated that HOTAIR relative expression in tumor and paired blood are positively correlated $(R=0.43, p=0.03)$. In univariate analysis, HOTAIR levels in blood were associated with higher mortality of patients (Cox's proportional hazard, hazard ratio $=5.9,95 \%$ confidence interval: $1.3-26.1$, 
$p=0.019$ ). This means that the HOTAIR expression in blood can also be an independent prognostic marker in CRC. As a kind of surrogate sample of tumor tissue, the non-invasively obtained HOTAIR data may have vast clinical potential in prognosis. Moreover, HOTAIR overexpression-either in tumor tissue or in blood-may identify patients that would require more intensive care of personally tailored treatment.

EZH2 and SUZ12, the components of PRC2, are overexpressed in several cancers. In particular, SUZ12 is reportedly overexpressed in colorectal cancer. Gene pathway analysis indicated that HOTAIR-regulated gene sets included CDH1 (E-cadherin) target genes, whose expression is lost in metastatic cancer cells of the mesenchymal phenotype. Thus, HOTAIR might cooperate with PRC2 to maintain mesenchymal and undifferentiated cancer cells. For these reasons, HOTAIR or SUZ12 might be a treatment target in colorectal cancer.

Studies of HOTAIR in gastric cancer (GC) have just begun, but its relevance has already been demonstrated. Using soft agar assays, Endo et al. [17] showed that the anchorage-independent growth of gastric cancer cells depended on HOTAIR expression. They grafted gastric cancer cells with increased or suppressed HOTAIR expression into the tail vein or peritoneal cavity of immunodeficient mice. The HOTAIR-expressing group showed greater venous invasion, more frequent lymph node metastases, and lower overall survival rate than the HOTAIR-suppressed group. HOTAIR-expressing cells were also more likely to induce liver metastases. These findings suggest that HOTAIR has prognostic value in gastric cancer in vitro and in vivo. However, the molecular mechanism of HOTAIR in gastric cancer is poorly understood. Recently published data suggested it may function as a miRNA sponge and induces down-regulation of $m i R-331-3 p$, thereby modulating the derepression of HER 2 expression and promoting migration and invasion of gastric cancer cells [44,45]. Meanwhile, $\mathrm{Xu}$ et al. [37] found that inhibition of HOTAIR could reduce invasiveness and reverse EMT process in these cells. These results also indicate that HOTAIR may be a therapeutic target in the treatment of gastric cancer.

\subsection{HOTAIR Is a Potential Prognostic Factor of Hepatoma Metastases and Relapse after Hepatic Resection or Liver Transplantation}

In primary hepatocellular carcinoma (HCC), HOTAIR overexpression is not observed in every cancer patient. However, a study of HCC patients showed that patients with HOTAIR overexpression exhibit larger tumor size, worse prognosis, and an increased risk of metastasis when compared to patients with normal HOTAIR levels [12]. In a study of 63 patients after hepatectomy, Geng et al. [46] found that HOTAIR was associated with hepatocellular growth and concluded that HOTAIR might be a biomarker for predicting lymph node metastasis. In some cancer patients, the HOTAIR level of the cancer biopsy was higher than adjacent normal tissue; and this cohort showed higher risk of relapse. Knockdown of HOTAIR down-regulated proteins related to cell motility and metastasis, such as matrix metalloproteinase (MMP)-9 and vascular endothelial growth factor (VEGF), and decreased proliferation of Bel7402, a hepatoma cell line. On the other hand, HOTAIR levels were increased in tumor samples from patients with lymph node metastasis.

In some patients who underwent liver transplantation, Yang et al. [31] also observed higher HOTAIR expression in cancer tissues than in adjacent normal tissues and found that HOTAIR was an 
independent prognostic factor for relapse $(p=0.001)$. The survival period after relapse was shorter in these patients. Similarly, in a hepatoma cell line, interfering HOTAIR function with specific siRNA affected viability and invasiveness and increased the sensitivity to TNF- $\alpha$-induced apoptosis and cisplatinum/doxorubicin treatment. Thus, after liver transplantation, HOTAIR is a promising prognostic factor and a potential target for treatment.

Recently, the research team of Yang reported their study on the role and molecular mechanism of HOTAIR in HCC progression. They found that HOTAIR suppression significantly increased the expression of RNA binding motif protein 38 (RBM38), and the expression levels of RBM38 were significantly lower than adjacent normal tissues. Moreover, RBM38 suppression by siRNA strategy also reversed the cell migration and invasion compared with HOTAIR-knockdown cells [47]. These results suggest that RBM38 is one of the direct target of HOTAIR in HCC progression, and underlines the potential of HOTAIR as a therapy target.

VEGF and MMP-9 play important roles in hepatocellular carcinoma progression. Because these proteins are regulated by HOTAIR, treatments that target HOTAIR might suppress growth. On the other hand, drugs that target VEGF or MMP-9 directly might also be helpful for hepatoma treatment.

In summary, the lncRNA HOTAIR is important in breast, gastroenteric, and liver cancers. The signaling molecules most likely to interact with HOTAIR in these cancers are shown in Table 2, and some of the related pathways that are most likely to be applied to therapeutic strategy are enumerated in Tables 3-6.

Table 2. HOTAIR-related signaling molecules.

\begin{tabular}{cccc}
\hline Cancer Type & IncRNA & Related Molecules & Reference \\
\hline BC & HOTAIR & PRC2/LSD1/E2/BRCA1 & {$[11,30]$} \\
CRC & HOTAIR & EZH2/SUZ12/CDH1 & {$[11,13]$} \\
HCC & HOTAIR & VEGF/MMP-9 & {$[31]$} \\
\hline
\end{tabular}

Table 3. HOTAIR-related signaling pathways/processes in BC.

\begin{tabular}{ccc}
\hline Pathways & Signaling Molecules & Biological Processes \\
\hline ErbB signaling pathway & HER2 & cell migration/evasion \\
\hline NF-kappa B signaling pathway & HER2 & DNA degradation/cell survival \\
\hline PI3K-Akt signaling pathway & BRCA1/EZH2 & DNA repair/cell proliferation; angiogenesis \\
\hline TGF- $\beta$ signaling pathway & twist/snail/miR-10b & EMT process; Cell growth/survival; \\
& E2 & Cell migration/invasion \\
\hline estrogen signaling pathway & BRCA1/E2 & Degradation of target protein \\
\hline ubiquitin mediated proteolysis & &
\end{tabular}

Table 4. HOTAIR-related signaling pathways/processes in CRC.

\begin{tabular}{ccc}
\hline Pathways & Signaling Molecules & Biological Processes \\
\hline TGF- $\beta$ signaling pathway & snail & EMT process \\
P53 signaling pathway & EZH2/CDK1 & Cell cycle arrest \\
Cell cycle & CDH1/EZH2/CDK1 & Cell cycle arrest \\
Ubiquitin mediated proteolysis & CDH1 & Degradation of target protein \\
\hline
\end{tabular}


Table 5. HOTAIR-related signaling pathways/processes in GC.

\begin{tabular}{ccc}
\hline Pathways & Signaling Molecules & Biological Processes \\
\hline TGF- $\beta$ signaling pathway & snail & EMT process \\
\hline RNA interfere & $m i R-331-3 p$ & miRNA sponge/HER2-mRNA protection; \\
& & cell signaling networks \\
\hline
\end{tabular}

Table 6. HOTAIR-related signaling pathways/processes in HCC.

\begin{tabular}{ccc}
\hline Pathways & Signaling Molecules & Biological Processes \\
\hline VEGF signaling pathway & VEGF & $\begin{array}{c}\text { cell proliferation/migration; } \\
\text { sustained angiogenesis }\end{array}$ \\
\hline TNF signaling pathway & MMP-3/MMP-9 & remodeling of extracellular matrix \\
\hline PPAR signaling pathway & MMP-1 & adipocyte differentiation \\
\hline Wnt signaling pathway & MMP-7 & cell cycle \\
\hline mRNA surveillance pathway & RBM38/p53 & gene overexpression \\
TGF- $\beta$ signaling pathway & $m i R-10 b$ & $\begin{array}{c}\text { EMT process; cell growth and survival; } \\
\text { cell migration and invasion }\end{array}$ \\
\hline
\end{tabular}

\subsection{Other HOTAIR-Related Cancers}

A relationship between HOTAIR and other types of tumors, including lung cancer, prostate cancer, pancreatic carcinoma, and sarcoma, has been reported. In non-small cell lung cancer, the effect of HOTAIR on cell invasion and metastases is induced by HOXA5 [48]. Loss of HOTAIR in non-small cell lung cancer cells led to a significant decrease in the levels of MMP-2 and MMP-9, which are important for many biology processes, including cell proliferation, differentiation, remodeling of the extracellular matrix, vascularization, and cell migration. In lung adenocarcinoma, HOTAIR overexpression and p21 are responsible for cisplatinum resistance [49]. Depletion of HOTAIR by siRNA led to decreased invasion and increased apoptosis in HepG2 cells, and tumor growth was significantly inhibited in mice injected with HOTAIR-deficient cells [50]. Overexpression of HOTAIR is also associated with high-grade tumor and metastasis in gastrointestinal stromal tumors; knockdown of HOTAIR altered the expression of some reported target genes and suppressed cell invasiveness [51].

\section{Conclusions}

HOTAIR is dysregulated in some cancers. It plays a role in tumorigenesis and tumor progression, and it has prognostic value in BC, CRC, and HCC. Treatments that target HOTAIR are a new area of tumor research. However, large-scale and multicenter randomized tests are needed to confirm the value of HOTAIR as a prognostic factor. Meanwhile, to develop therapeutic applications with HOTAIR as a target, the pathways and molecules downstream of HOTAIR must be elucidated. Research into HOTAIR will hopefully lead to theranostic applications in human cancer.

Anti-tumor strategies that focus on RNA as a target molecule are currently under development. For cancer therapy, the cancer- and tissue-specific expression of lncRNAs is an advantage not offered by other therapeutic options. The use of HOTAIR in cancer therapy will require detailed knowledge about tumor-specific ncRNA function and cancer cell properties. 
Theoretically, HOTAIR activity could be blocked in multiple ways. First, one could block molecular interactions using small molecule inhibitors that mask the binding site in protein interaction partners or antagonistic oligonucleotides that bind to the ncRNA and interfere with protein binding, thus silencing HOTAIR. Second, one could disrupt the secondary structure of HOTAIR using small molecule inhibitors or mimics to compete at the binding site. Third, HOTAIR could be silenced with specific siRNAs or redundant miRNAs. In addition to blocking HOTAIR, drugs could be developed that directly target molecules in the HOTAIR pathway listed in Tables 3-6, such as ERs in BC, SUZ12 in CRC, and VEGF/MMP-9 in HCC. Meanwhile, further research into the HOTAIR pathway and related molecules is needed. HOTAIR shows great prognostic and therapeutic promise for various kinds of cancer.

\section{Author Contributions}

All authors have contributed significantly and equally to this work.

\section{Conflicts of Interest}

The authors declare no conflict of interest.

\section{References}

1. International Human Genome Sequencing Consortium. Finishing the euchromatic sequence of the human genome. Nature 2004, 431, 931-945.

2. Gutschner, T.; Diederichs, S. The hallmarks of cancer: A long non-coding rna point of view. RNA Biol. 2012, 9, 703-719.

3. Ponting, C.P.; Oliver, P.L.; Reik, W. Evolution and functions of long noncoding rnas. Cell 2009, 136, 629-641.

4. Rinn, J.L.; Kertesz, M.; Wang, J.K.; Squazzo, S.L.; Xu, X.; Brugmann, S.A.; Goodnough, L.H.; Helms, J.A.; Farnham, P.J.; Segal, E.; et al. Functional demarcation of active and silent chromatin domains in human hox loci by noncoding rnas. Cell 2007, 129, 1311-1323.

5. Tano, K.; Mizuno, R.; Okada, T.; Rakwal, R.; Shibato, J.; Masuo, Y.; Ijiri, K.; Akimitsu, N. Malat-1 enhances cell motility of lung adenocarcinoma cells by influencing the expression of motility-related genes. FEBS Lett. 2010, 584, 4575-4580.

6. Xu, C.; Yang, M.; Tian, J.; Wang, X.; Li, Z. Malat-1: A long non-coding rna and its important 3' end functional motif in colorectal cancer metastasis. Int. J. Oncol. 2011, 39, 169-175.

7. Ji, Q.; Liu, X.; Fu, X.; Zhang, L.; Sui, H.; Zhou, L.; Sun, J.; Cai, J.; Qin, J.; Ren, J.; et al. Resveratrol inhibits invasion and metastasis of colorectal cancer cells via malatl mediated wnt/beta-catenin signal pathway. PLoS One 2013, 8, e78700.

8. Lai, M.C.; Yang, Z.; Zhou, L.; Zhu, Q.Q.; Xie, H.Y.; Zhang, F.; Wu, L.M.; Chen, L.M.; Zheng, S.S. Long non-coding rna malat-1 overexpression predicts tumor recurrence of hepatocellular carcinoma after liver transplantation. Med. Oncol. 2012, 29, 1810-1816.

9. Lin, R.; Maeda, S.; Liu, C.; Karin, M.; Edgington, T.S. A large noncoding rna is a marker for murine hepatocellular carcinomas and a spectrum of human carcinomas. Oncogene 2007, 26, 851-858. 
10. Yang, L.; Lin, C.; Liu, W.; Zhang, J.; Ohgi, K.A.; Grinstein, J.D.; Dorrestein, P.C.; Rosenfeld, M.G. Ncrna- and pc2 methylation-dependent gene relocation between nuclear structures mediates gene activation programs. Cell 2011, 147, 773-788.

11. Gupta, R.A.; Shah, N.; Wang, K.C.; Kim, J.; Horlings, H.M.; Wong, D.J.; Tsai, M.-C.; Hung, T.; Argani, P.; Rinn, J.L. Long non-coding rna hotair reprograms chromatin state to promote cancer metastasis. Nature 2010, 464, 1071-1076.

12. Ishibashi, M.; Kogo, R.; Shibata, K.; Sawada, G.; Takahashi, Y.; Kurashige, J.; Akiyoshi, S.; Sasaki, S.; Iwaya, T.; Sudo, T., et al. Clinical significance of the expression of long non-coding rna hotair in primary hepatocellular carcinoma. Oncol. Rep. 2013, 29, 946-950.

13. Kogo, R.; Shimamura, T.; Mimori, K.; Kawahara, K.; Imoto, S.; Sudo, T.; Tanaka, F.; Shibata, K.; Suzuki, A.; Komune, S. Long noncoding rna hotair regulates polycomb-dependent chromatin modification and is associated with poor prognosis in colorectal cancers. Cancer Res. 2011, 71, 6320-6326.

14. Nie, Y.; Liu, X.; Qu, S.; Song, E.; Zou, H.; Gong, C. Long non-coding rna hotair is an independent prognostic marker for nasopharyngeal carcinoma progression and survival. Cancer Sci. 2013, 104, 458-464.

15. Sorensen, K.P.; Thomassen, M.; Tan, Q.; Bak, M.; Cold, S.; Burton, M.; Larsen, M.J.; Kruse, T.A. Long non-coding rna hotair is an independent prognostic marker of metastasis in estrogen receptor-positive primary breast cancer. Breast Cancer Res. Treat. 2013, 142, 529-536.

16. Chen, F.J.; Sun, M.; Li, S.Q.; Wu, Q.Q.; Ji, L.; Liu, Z.L.; Zhou, G.Z.; Cao, G.; Jin, L.; Xie, H.W.; et al. Upregulation of the long non-coding rna hotair promotes esophageal squamous cell carcinoma metastasis and poor prognosis. Mol. Carcinog. 2013, 52, 908-915.

17. Endo, H.; Shiroki, T.; Nakagawa, T.; Yokoyama, M.; Tamai, K.; Yamanami, H.; Fujiya, T.; Sato, I.; Yamaguchi, K.; Tanaka, N. Enhanced expression of long non-coding rna hotair is associated with the development of gastric cancer. PLoS One 2013, 8, e77070.

18. Hajjari, M.; Behmanesh, M.; Sadeghizadeh, M.; Zeinoddini, M. Up-regulation of hotair long non-coding RNA in human gastric adenocarcinoma tissues. Med. Oncol. 2013, 30, 670.

19. Kim, K.; Jutooru, I.; Chadalapaka, G.; Johnson, G.; Frank, J.; Burghardt, R.; Kim, S.; Safe, S. Hotair is a negative prognostic factor and exhibits pro-oncogenic activity in pancreatic cancer. Oncogene 2013, 32, 1616-1625.

20. Zhang, J.X.; Han, L.; Bao, Z.S.; Wang, Y.Y.; Chen, L.Y.; Yan, W.; Yu, S.Z.; Pu, P.Y.; Liu, N.; You, Y.P.; et al. Hotair, a cell cycle-associated long noncoding RNA and a strong predictor of survival, is preferentially expressed in classical and mesenchymal glioma. Neuro Oncol. 2013, 15, 1595-1603.

21. Zhang, J.; Zhang, P.; Wang, L.; Piao, H.L.; Ma, L. Long non-coding RNA hotair in carcinogenesis and metastasis. Acta Biochim. Biophys. Sin. 2014, 46, 1-5.

22. Panzitt, K.; Tschernatsch, M.M.; Guelly, C.; Moustafa, T.; Stradner, M.; Strohmaier, H.M.; Buck, C.R.; Denk, H.; Schroeder, R.; Trauner, M.; et al. Characterization of hulc, a novel gene with striking up-regulation in hepatocellular carcinoma, as noncoding RNA. Gastroenterology 2007, 132, 330-342.

23. Matouk, I.J.; Abbasi, I.; Hochberg, A.; Galun, E.; Dweik, H.; Akkawi, M. Highly upregulated in liver cancer noncoding RNA is overexpressed in hepatic colorectal metastasis. Eur. J. Gastroenterol. Hepatol. 2009, 21, 688-692. 
24. Wang, J.; Liu, X.; Wu, H.; Ni, P.; Gu, Z.; Qiao, Y.; Chen, N.; Sun, F.; Fan, Q. Creb up-regulates long non-coding RNA, hulc expression through interaction with microrna-372 in liver cancer. Nucleic Acids Res. 2010, 38, 5366-5383.

25. Matouk, I.J.; DeGroot, N.; Mezan, S.; Ayesh, S.; Abu-lail, R.; Hochberg, A.; Galun, E. The $h 19$ non-coding RNA is essential for human tumor growth. PLoS One 2007, 2, e845.

26. Pasmant, E.; Laurendeau, I.; Heron, D.; Vidaud, M.; Vidaud, D.; Bieche, I. Characterization of a germ-line deletion, including the entire ink4/arf locus, in a melanoma-neural system tumor family: Identification of anril, an antisense noncoding RNA whose expression coclusters with arf. Cancer Res. 2007, 67, 3963-3969.

27. Turnbull, C.; Ahmed, S.; Morrison, J.; Pernet, D.; Renwick, A.; Maranian, M.; Seal, S.; Ghoussaini, M.; Hines, S.; Healey, C.S.; et al. Genome-wide association study identifies five new breast cancer susceptibility loci. Nat. Genet. 2010, 42, 504-507.

28. Yap, K.L.; Li, S.; Munoz-Cabello, A.M.; Raguz, S.; Zeng, L.; Mujtaba, S.; Gil, J.; Walsh, M.J.; Zhou, M.M. Molecular interplay of the noncoding rna anril and methylated histone h3 lysine 27 by polycomb cbx7 in transcriptional silencing of ink4a. Mol. Cell 2010, 38, 662-674.

29. Kotake, Y.; Nakagawa, T.; Kitagawa, K.; Suzuki, S.; Liu, N.; Kitagawa, M.; Xiong, Y. Long non-coding RNA anril is required for the prc2 recruitment to and silencing of p15(ink4b) tumor suppressor gene. Oncogene 2011, 30, 1956-1962.

30. Tsai, M.C.; Manor, O.; Wan, Y.; Mosammaparast, N.; Wang, J.K.; Lan, F.; Shi, Y.; Segal, E.; Chang, H.Y. Long noncoding RNA as modular scaffold of histone modification complexes. Science 2010, 329, 689-693.

31. Yang, Z.; Zhou, L.; Wu, L.M.; Lai, M.C.; Xie, H.Y.; Zhang, F.; Zheng, S.S. Overexpression of long non-coding RNA hotair predicts tumor recurrence in hepatocellular carcinoma patients following liver transplantation. Ann. Surg. Oncol. 2011, 18, 1243-1250.

32. Tano, K.; Akimitsu, N. Long non-coding RNAs in cancer progression. Front. Genet. 2012, 3, 219.

33. He, S.; Liu, S.; Zhu, H. The sequence, structure and evolutionary features of hotair in mammals. BMC Evolut. Biol. 2011, 11, 102.

34. Kaneko, S.; Li, G.; Son, J.; Xu, C.F.; Margueron, R.; Neubert, T.A.; Reinberg, D. Phosphorylation of the prc2 component ezh2 is cell cycle-regulated and up-regulates its binding to ncrna. Genes Dev. 2010, 24, 2615-2620.

35. Schorderet, P.; Duboule, D. Structural and functional differences in the long non-coding RNA hotair in mouse and human. PLoS Genet. 2011, 7, e1002071.

36. Chiyomaru, T.; Yamamura, S.; Fukuhara, S.; Yoshino, H.; Kinoshita, T.; Majid, S.; Saini, S.; Chang, I.; Tanaka, Y.; Enokida, H.; et al. Genistein inhibits prostate cancer cell growth by targeting mir-34a and oncogenic hotair. PLoS One 2013, 8, e70372.

37. Xu, Z.Y.; Yu, Q.M.; Du, Y.A.; Yang, L.T.; Dong, R.Z.; Huang, L.; Yu, P.F.; Cheng, X.D. Knockdown of long non-coding RNA hotair suppresses tumor invasion and reverses epithelial-mesenchymal transition in gastric cancer. Int. J. Biol. Sci. 2013, 9, 587-597.

38. Padua Alves, C.; Fonseca, A.S.; Muys, B.R.; de Barros, E.L.B.R.; Burger, M.C.; de Souza, J.E.; Valente, V.; Zago, M.A.; Silva, W.A., Jr. Brief report: The lincrna hotair is required for epithelialto-mesenchymal transition and stemness maintenance of cancer cells lines. Stem Cells 2013, 31, $2827-2832$. 
39. Jalali, S.; Bhartiya, D.; Lalwani, M.K.; Sivasubbu, S.; Scaria, V. Systematic transcriptome wide analysis of lncrna-mirna interactions. PLoS One 2013, 8, e53823.

40. Wang, L.; Zeng, X.; Chen, S.; Ding, L.; Zhong, J.; Zhao, J.C.; Wang, L.; Sarver, A.; Koller, A.; Zhi, J.; et al. Brcal is a negative modulator of the prc2 complex. EMBOJ. 2013, 32, 1584-1597.

41. Lu, L.; Zhu, G.; Zhang, C.; Deng, Q.; Katsaros, D.; Mayne, S.T.; Risch, H.A.; Mu, L.; Canuto, E.M.; Gregori, G.; et al. Association of large noncoding RNA hotair expression and its downstream intergenic cpg island methylation with survival in breast cancer. Breast Cancer Res. Treat. 2012, $136,875-883$.

42. Bhan, A.; Hussain, I.; Ansari, K.I.; Kasiri, S.; Bashyal, A.; Mandal, S.S. Antisense transcript long noncoding RNA (lncrna) hotair is transcriptionally induced by estradiol. J. Mol. Biol. 2013, 425, 3707-3722.

43. Svoboda, M.; Slyskova, J.; Schneiderova, M.; Makovicky, P.; Bielik, L.; Levy, M.; Lipska, L.; Hemmelova, B.; Kala, Z.; Protivankova, M., et al. Hotair long non-coding RNA is a negative prognostic factor not only in primary tumors, but also in the blood of colorectal cancer patients. Carcinogenesis 2014, 35, 1510-1515.

44. Liu, X.H.; Sun, M.; Nie, F.Q.; Ge, Y.B.; Zhang, E.B.; Yin, D.D.; Kong, R.; Xia, R.; Lu, K.H.; Li, J.H.; et al. Lnc RNA hotair functions as a competing endogenous rna to regulate her2 expression by sponging mir-331-3p in gastric cancer. Mol. Cancer 2014, 13, 92.

45. Lee, N.K.; Lee, J.H.; Park, C.H.; Yu, D.; Lee, Y.C.; Cheong, J.H.; Noh, S.H.; Lee, S.K. Long non-coding RNA hotair promotes carcinogenesis and invasion of gastric adenocarcinoma. Biochem. Biophys. Res. Commun. 2014, 451, 171-178.

46. Geng, Y.J.; Xie, S.L.; Li, Q.; Ma, J.; Wang, G.Y. Large intervening non-coding rna hotair is associated with hepatocellular carcinoma progression. J. Int. Med. Res. 2011, 39, 2119-2128.

47. Ding, C.; Cheng, S.; Yang, Z.; Lv, Z.; Xiao, H.; Du, C.; Peng, C.; Xie, H.; Zhou, L.; Wu, J.; et al. Long non-coding RNA hotair promotes cell migration and invasion via down-regulation of RNA binding motif protein 38 in hepatocellular carcinoma cells. Int. J. Mol. Sci. 2014, 15, 4060-4076.

48. Liu, X.H.; Liu, Z.L.; Sun, M.; Liu, J.; Wang, Z.X.; De, W. The long non-coding RNA hotair indicates a poor prognosis and promotes metastasis in non-small cell lung cancer. BMC Cancer 2013, 13, 464.

49. Liu, Z.; Sun, M.; Lu, K.; Liu, J.; Zhang, M.; Wu, W.; De, W.; Wang, Z.; Wang, R. The long noncoding rna hotair contributes to cisplatin resistance of human lung adenocarcinoma cells via downregualtion of $p 21$ (waf1/cip1) expression. PLoS One 2013, 8, e77293.

50. Li, D.; Feng, J.; Wu, T.; Wang, Y.; Sun, Y.; Ren, J.; Liu, M. Long intergenic noncoding RNA hotair is overexpressed and regulates pten methylation in laryngeal squamous cell carcinoma. Am. J. Pathol. 2013, 182, 64-70.

51. Niinuma, T.; Suzuki, H.; Nojima, M.; Nosho, K.; Yamamoto, H.; Takamaru, H.; Yamamoto, E.; Maruyama, R.; Nobuoka, T.; Miyazaki, Y.; et al. Upregulation of mir-196a and hotair drive malignant character in gastrointestinal stromal tumors. Cancer Res. 2012, 72, 1126-1136.

(C) 2014 by the authors; licensee MDPI, Basel, Switzerland. This article is an open access article distributed under the terms and conditions of the Creative Commons Attribution license (http://creativecommons.org/licenses/by/4.0/). 